\title{
Higgs-to-Invisible Searches with the CMS experiment at the LHC
}

\author{
Riccardo Di Maria*, on behalf of the CMS Collaboration \\ Imperial College London, UK \\ E-mail: riccardo.di.mariadcern.ch
}

\begin{abstract}
Searches for invisible decays of the Higgs boson are presented using data at a centre-of-mass energy of $\sqrt{s}=13 \mathrm{TeV}$ collected with the CMS detector at the LHC in 2016. The dataset corresponds to an integrated luminosity of $35.9 \mathrm{fb}^{-1}$. The search channel targets Higgs boson production via vector boson fusion. The results are presented in terms of an upper limit on the branching fraction of the Higgs boson decay to invisible particles $\mathscr{B}(H \rightarrow$ inv. $)$. The data are in agreement with the contribution of backgrounds from standard model processes. An observed (expected) upper limit on $\mathscr{B}(H \rightarrow$ inv. $)$ at $95 \%$ confidence level is set at $0.53(0.27)$ for the cut-and-count and at $0.28(0.21)$ for the shape approaches, respectively. A combination with other relevant analyses to further improve the sensitivity to $\mathscr{B}(H \rightarrow$ inv. $)$ is also presented using the 2016 dataset. An observed (expected) upper limit on $\mathscr{B}(H \rightarrow$ inv.) at 95\% confidence level is set at $0.24(0.18)$, assuming standard model production rates. This result represents the most sensitive Higgs-toinvisible search, and it is interpreted in the context of Higgs-portal models for dark matter where upper bounds are placed on the dark matter-nucleon spin-independent cross-section.
\end{abstract}

Sixth Annual Conference on Large Hadron Collider Physics (LHCP2018)

4-9 June 2018

Bologna, Italy

\footnotetext{
* Speaker.
} 


\section{Introduction}

The discovery of the Higgs boson at the Large Hadron Collider (LHC) during Run-1, along with the absence of any significant experimental evidence for physics beyond the standard model, have placed constraints on new physics models. Although the observed $125 \mathrm{GeV}$ boson is compatible with the standard model (SM) Higgs boson, the existence of non-SM properties is not presently excluded due to the associated uncertainties. The possibility for further Higgs bosons with exotic decay modes remains, and invisible decay modes could be possible in interactions between the Higgs boson and dark matter, where the former becomes the mediator between SM and dark matter particles.

A Higgs boson decaying to invisible particles cannot be observed directly [1]. In order to identify such an event, the Higgs boson has to be produced with other particles, and the missing transverse energy ( $E_{\mathrm{T}}^{\text {miss }}$ or MET) can be exploited to carry out these searches. The vector boson fusion (VBF) channel is the most sensitive due to its characteristic topology and it has a large cross-section in comparison with the other production modes [2]. The VBF jets are produced with a large separation in pseudo-rapidity, large invariant mass $\left(m_{\mathrm{jj}}\right)$, and small azimuthal separation due to kinematic considerations, and the absence of colour connection between them. The pseudo-

rapidity is defined as $\eta=-\ln \left(\tan \frac{\theta}{2}\right)$, where $\theta$ is the polar angle measured from the $z$-axis in the longitudinal plane $y-z$.

These proceedings describe the search for invisibly decaying Higgs bosons produced through VBF mode using the 2016 data at a centre-of-mass energy of $\sqrt{s}=13 \mathrm{TeV}$. The data have been collected with the CMS detector, a multi-purpose apparatus designed to study a wide range of physics processes. A detailed description of the CMS detector and of the relevant kinematic variables, together with a definition of the coordinate system used, can be found in Ref. [3]. The 2016 analysis builds on the analyses performed with the 2012 data [4], parked-data [5] and 2015 data [6], all contained in Ref. [7]. Several improvements compared to the 2015 analysis are detailed in these proceedings, e.g. new control samples in data thereby further improving the precision of the background estimation. A combination with other relevant analyses targeting the other main production modes of the Higgs boson is also presented, to further improve the sensitivity of the branching fraction of the Higgs boson decay to invisible particles $\mathscr{B}(H \rightarrow$ inv. $)$.

\section{Simulated Samples, Triggers, and Physics Objects}

The experimental signature of this analysis consists of two VBF jets and sizeable missing transverse energy coming from the invisible Higgs boson decay. However, there are several SM backgrounds to the VBF $H \rightarrow$ inv. signal. Simulated events are passed as input to a GEANT4 [8] based simulation of the CMS detector. The processes considered are as follows: $Z(v \bar{v})+$ jets, $Z(l \bar{l})+$ jets (where $l$ is either an electron or a muon), $W+$ jets, Top-quark decays (both $t \bar{t}$ and single top), Diboson (WW, WZ, ZZ) decays, and QCD multijet. Jets are reconstructed by clustering particle-flow (PF) candidates using the anti- $\mathrm{k}_{\mathrm{T}}$ algorithm with a distance parameter of 0.4 [9].

The data used for the analysis are collected using triggers designed to select events that have large $E_{\mathrm{T}}^{\text {miss }}$ and large missing transverse momentum calculated from jets $\left(H_{\mathrm{T}}^{\text {miss }}\right)$. The $E_{\mathrm{T}}^{\text {miss }}$ is computed as the magnitude of the negative vector sum of the transverse momenta of all PF [10] 
candidates reconstructed in a given event. Both the $E_{\mathrm{T}}^{\text {miss }}$ and $H_{\mathrm{T}}^{\text {miss }}$ quantities are computed without including muon candidates, allowing one to select dimuon (mainly $Z \rightarrow \mu \bar{\mu}$ ) and single muon (mainly $W \rightarrow \mu \bar{v}$ ) events in two different muon control regions (CRs). The hadronic recoil $\vec{U}$ is given by $\vec{U}=\vec{E}_{\mathrm{T}}^{\text {miss }}+\vec{p}_{\mathrm{T}}^{\text {di-lll, }}$, where the last $\vec{p}_{\mathrm{T}}$ term is the di-lepton or lepton transverse momentum, depending on the CR. Two additional CRs for this analysis include dielectron $(Z \rightarrow e \bar{e})$ and single electron $(W \rightarrow e \bar{v})$ events which are selected using a different trigger.

Several primary vertices are typically reconstructed in an event because of pileup. A loose set of selection criteria is applied to select well-reconstructed primary vertices and the vertex with the highest $p_{\mathrm{T}}$ sum of the constituent tracks is chosen as the one corresponding to the hard interaction.

\section{Selection}

The search presented in these proceedings has been performed using two different approaches. The shape analysis exploits distinctive kinematic features of the VBF topology by fitting the shape of the $m_{\mathrm{jj}}$ distribution. It provides the best expected sensitivity to invisible decays of the SM Higgs boson. The cut-and-count analysis allows for a re-interpretation of the results in the context of different phenomenological models targeting the same topology. Both approaches are optimised in terms of the expected 95\% confidence level limit on the branching ratio of the SM Higgs boson (with a mass of $125 \mathrm{GeV})$ decaying invisibly $\mathscr{B}(H \rightarrow$ inv.). The selection is driven by three considerations: the chosen trigger drives the $E_{\mathrm{T}}^{\text {miss }}$ criteria to avoid the sharp turn-on region of the trigger and be above the $90 \%$ efficiency point; the VBF topology of the jets expected from the signal; the rejection of the multijet background from which large $E_{\mathrm{T}}^{\text {miss }}$ can arise due to mis-measurements. The selection criteria for the signal region (SR) for both the shape and the cut-and-count analyses are summarised in Table 1.

Table 1: Summary of the kinematic selections used to define the SR for both the shape and the cut-and-count analyses [11].

\begin{tabular}{|c|c|c|c|}
\hline Observable & hape analysis & Cut-and-count analysis & Target background \\
\hline & \multicolumn{2}{|c|}{$\bar{p}_{\mathrm{T}}>80(40) \mathrm{GeV},|\eta|<4.7$} & All \\
\hline & \multirow{2}{*}{\multicolumn{2}{|c|}{$\begin{aligned} & >250 \mathrm{GeV} \\
& >0.5 \mathrm{rad}\end{aligned}$}} & QCD multijet, $\mathrm{t} \bar{t}, W+$ jets \\
\hline$\left.\vec{U}, \vec{p}_{\mathrm{T}}^{\mathrm{jets}}\right)$ & & & $\mathrm{QCD}_{\mathrm{i}}$ \\
\hline & \multirow{2}{*}{\multicolumn{2}{|c|}{$\begin{array}{c}\mathrm{N}_{\mu, \mathrm{e}}=0 \text { with } p_{\mathrm{T}}>10 \mathrm{GeV},|\eta|<2.4(2.5) \\
\mathrm{N}_{\tau_{\mathrm{H}}}=0 \text { with } p_{\mathrm{T}}>18 \mathrm{GeV},|\eta|<2.3\end{array}$}} & $W+$. \\
\hline & & & $W+$ jets, $Z(l \bar{l})+$ jets \\
\hline & \multirow{2}{*}{\multicolumn{2}{|c|}{$\begin{array}{c}\mathrm{N}_{\gamma}=0 \text { with } p_{\mathrm{T}}>15 \mathrm{GeV},|\eta|<2.5 \\
\mathrm{~N}_{\mathrm{jets}}=0 \text { with } p_{\mathrm{T}}>20 \mathrm{GeV}, \mathrm{CSV} 2>0.848\end{array}$}} & $\gamma+$ jets, $\mathrm{V} \gamma$ \\
\hline & & & $\mathrm{t} \overline{\mathrm{t}}$, single top quark \\
\hline$\eta_{\mathrm{j} 1} \cdot \eta_{\mathrm{j} 2}$ & \multirow{2}{*}{\multicolumn{2}{|c|}{$\begin{aligned} & <0 \\
< & <1.5 \mathrm{rad}\end{aligned}$}} & $Z(v \bar{v})+$ jets, $W(l \bar{v})+$ jets \\
\hline$\left|\Delta \phi_{\mathrm{jj}}\right|$ & & & $Z(v \bar{v})+$ jets, $W(l \bar{v})+$ jets \\
\hline$\left|\Delta \eta_{\mathrm{jj}}\right|$ & $>$ & $>4$ & $Z(v \bar{v})+$ jets, $W(l \bar{v})+$ jets \\
\hline$m_{\mathrm{jj}}$ & $>200 \mathrm{GeV}$ & $>1300 \mathrm{GeV}$ & $Z(v \bar{v})+$ jets, $W(l \bar{v})+$ jets \\
\hline
\end{tabular}

The distributions of $m_{\mathrm{jj}}$ for the shape analysis and $\Delta \eta_{\mathrm{jj}}$ after the cut-based selection are shown in Fig. 1. 

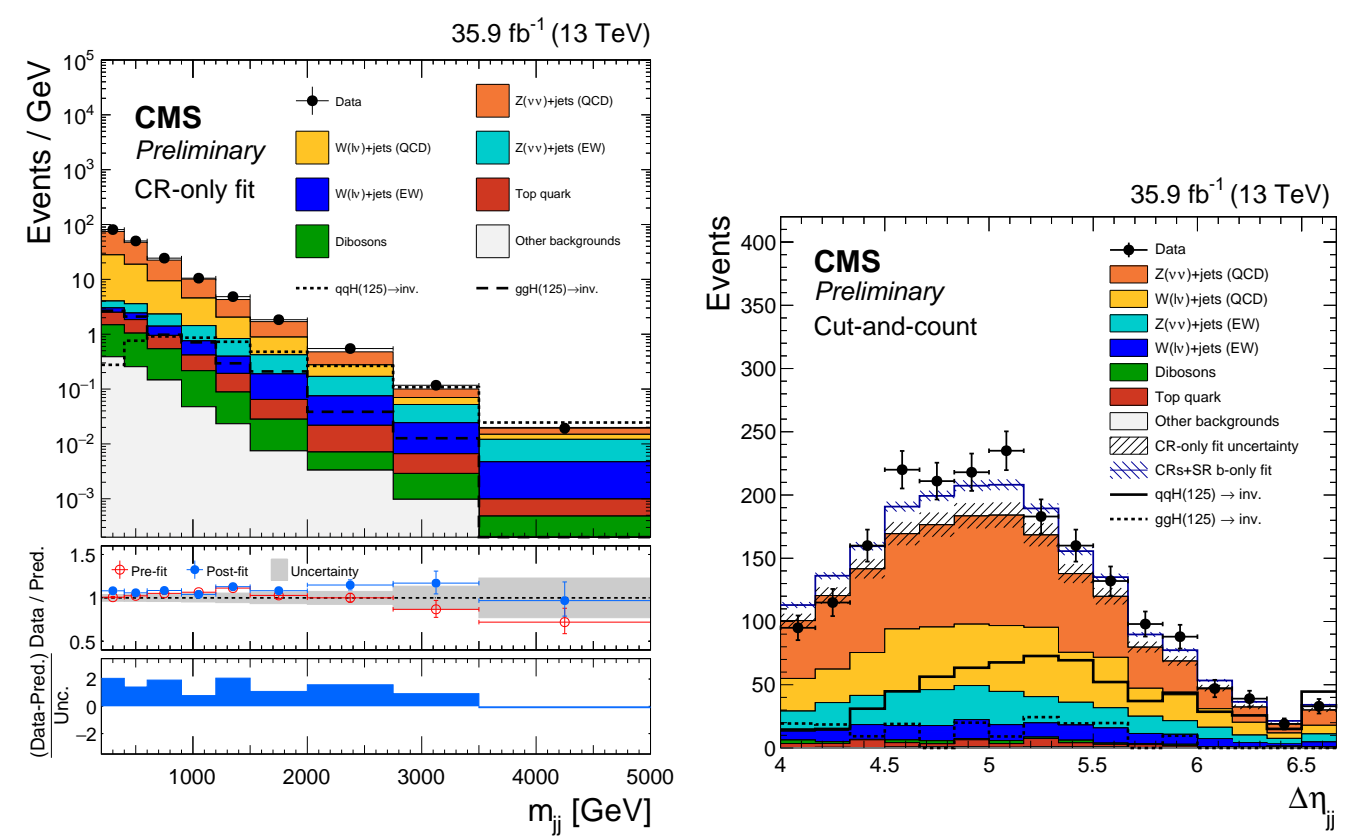

Figure 1: The observed $m_{\mathrm{jj}}$ distribution in the signal region compared to the post-fit backgrounds from various SM processes for the shape analysis obtained from a combined fit to the data in all the CRs, but excluding the SR (left). The observed $\Delta \eta_{\mathrm{jj}}$ distribution in the signal region compared to the post-fit backgrounds from various SM processes for the cut-and-count approach (right) [11].

\section{Control Regions}

Several background processes can mimic the signature of signal events. A large amount of $E_{\mathrm{T}}^{\mathrm{miss}}$ arises from $\mathrm{Z}$ bosons decaying to neutrinos, or from leptonic decays of $\mathrm{W}$ bosons where the lepton in the final state is missed. The $E_{\mathrm{T}}^{\mathrm{miss}}$ in the SR is estimated by constructing CRs that select either a single lepton or a pair of same flavour leptons. These correspond to regions where either $\mathrm{W}$ boson or $\mathrm{Z}$ boson production is dominant. Electrons and muons are thus used to define four CRs ( $W \rightarrow e \bar{v}$ and $Z \rightarrow e \bar{e}, W \rightarrow \mu \bar{v}$ and $Z \rightarrow \mu \bar{\mu}$ ) for estimating the key backgrounds ( $V+$ jets) in a data-driven approach. In this regard, the recoil in the CRs can be used to mimic the $E_{\mathrm{T}}^{\text {miss }}$ in $Z \rightarrow v \bar{v}$ events that fulfill the signal selection. As a result, the CRs are used to perform a simultaneous maximum likelihood fit to the data for the extraction of the final result. The distribution of $m_{\mathrm{jj}}$ in the single muon and dielectron CRs is shown in Fig. 2 for the cut-and-count analysis.

\section{Fit Procedure and Results}

A simultaneous fit to all the CRs and SR is performed using the 2016 dataset. The $V+$ jets normalisation relies on one parameter that ties together $\mathrm{W}$ and $\mathrm{Z}$ processes, and along with $\mathrm{QCD}$ and signal processes are free parameters in the fit. Moreover, corrections are applied to the $V+$ jets simulation to account for higher-order QCD and EWK effects on the ratios of $W+$ jets to $Z+$ jets differential cross-sections. The results of the simultaneous fit are interpreted as an upper limit 

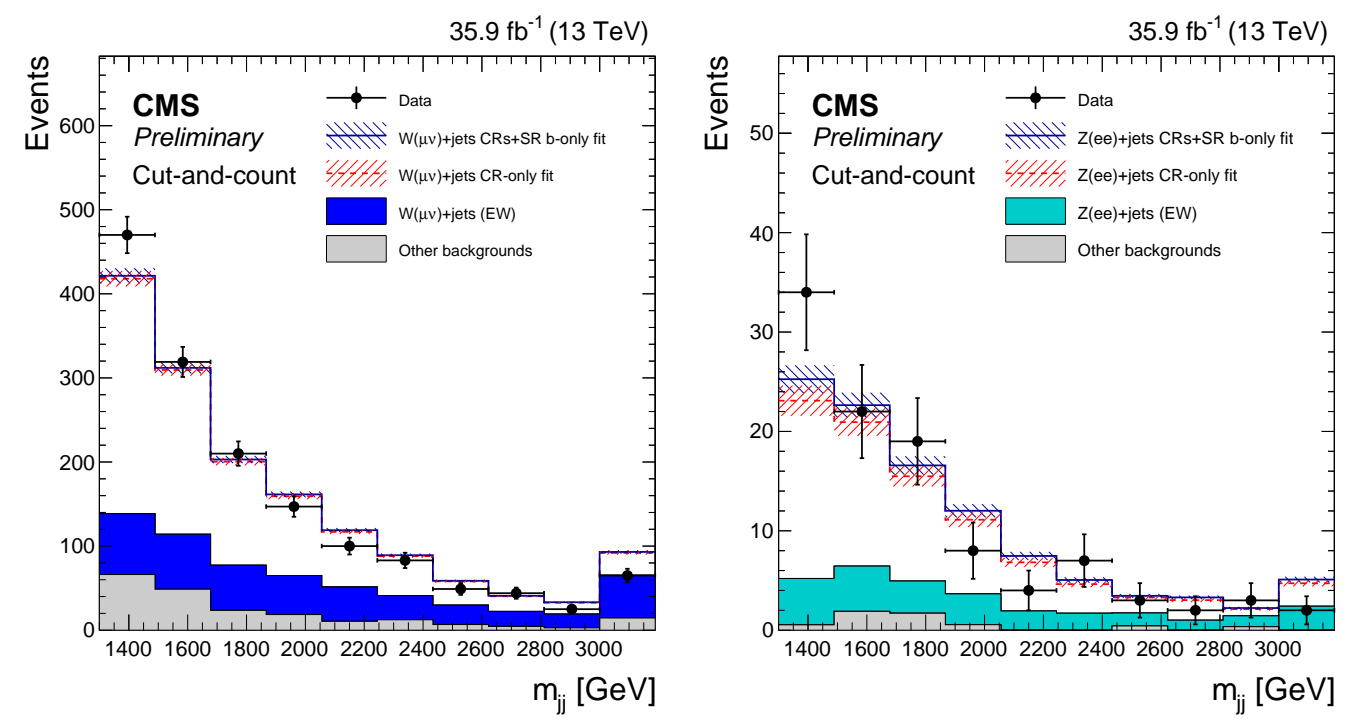

Figure 2: The observed $m_{\mathrm{jj}}$ distribution in the single muon (left) and dielectron (right) control regions compared to the post-fit backgrounds from various SM processes for the cut-and-count approach [11].

on the production cross-section times branching fraction for an invisibly decaying Higgs boson normalised to the SM VBF Higgs boson cross-section. An observed (expected) upper limit on $\mathscr{B}(H \rightarrow$ inv. $)$ at $95 \%$ confidence level is set at $0.53(0.27)$ for the cut-and-count and at $0.28(0.21)$ for the shape approaches respectively [11].

A combination with other relevant analyses to further improve the sensitivity to the branching fraction for an invisibly decaying Higgs boson is also performed using the 2016 dataset, and an observed (expected) upper limit on $\mathscr{B}(H \rightarrow$ inv. $)$ at $95 \%$ confidence level is set at $0.24(0.18)$, assuming standard model production rates [11]. This result represents the most sensitive Higgs-toinvisible search, and it can be translated into dark matter-nucleon spin-independent cross-section limits as a function of dark matter mass, using Higgs-portal models and assuming scalar and fermion dark matter candidates. 


\section{References}

[1] Higgs Working Group (Cavalli, D. et al.), The Higgs Working Group: Summary Report, hep-ph/0203056 FERMILAB-CONF-01-477.

[2] Y. Bai, P. Draper, J. Shelton, Measuring the Invisible Higgs Width at the 7 and 8 TeV LHC, JHEP 1207 (2012) 192.

[3] The CMS Collaboration, The CMS experiment at the CERN LHC, JINST 3 (2008) S08004, doi:10.1088/1748-0221/3/08/S08004.

[4] The CMS Collaboration, Search for invisible decays of Higgs bosons in the vector boson fusion and associated ZH production modes, Eur. Phys. J. C 74 (2014) 2980.

[5] The CMS Collaboration, Search for invisible decays of Higgs bosons in the vector boson fusion production mode, CMS Physics Analysis Summary HIG-14-038 (2015), URL: http://cds.cern.ch/record/2007270.

[6] The CMS Collaboration, Search for invisible decays of a Higgs boson produced via vector boson fusion at $\sqrt{s}=13 \mathrm{TeV}$, CMS Physics Analysis Summary HIG-16-009 (2016), URL: https://cds.cern.ch/record/2142460.

[7] The CMS Collaboration, Searches for invisible decays of the Higgs boson in pp collisions at $\sqrt{s}=7$, 8 , and $13 \mathrm{TeV}$, JHEP 02 (2017) 135.

[8] S. Agostinelli et al., Nuclear Instruments and Methods in Physics Research, A 506 (2003) 250.

[9] M. Cacciari, G. P. Salam, G. Soyez, The Anti-k(t) jet clustering algorithm, JHEP 0804 (2008) 063.

[10] The CMS Collaboration, Particle-flow reconstruction and global event description with the CMS detector, JINST 12 (2017) P10003, doi:10.1088/1748-0221/12/10/P10003, arXiv:1706.04965.

[11] The CMS Collaboration, Search for invisible decays of the Higgs boson produced through vector boson fusion at $\sqrt{s}=13 \mathrm{TeV}$, CMS Physics Analysis Summary HIG-17-023 (2018), URL: https://cds.cern.ch/record/2308434. 